\title{
Associations between Systemic Sclerosis and Thyroid Diseases
}

\author{
Poupak Fallahi' ${ }^{1 *}$, llaria Ruffilli', Dilia Giuggioli ${ }^{2}$, Michele Colaci ${ }^{2}$, Silvia Martina Ferrari ${ }^{1}$, \\ Alessandro Antonelli' and Clodoveo Ferri ${ }^{2}$ \\ ${ }^{1}$ Department of Clinical and Experimental Medicine, University of Pisa, Pisa, Italy, ${ }^{2}$ Department of Medical, Surgical, \\ Maternal, Pediatric and Adult Sciences, University of Modena and Reggio Emilia, Modena, Italy
}

\section{OPEN ACCESS}

Edited by:

Alex Stewart Stagnaro-Green,

University of llinois Rockford

College of Medicine, United States

Reviewed by:

Angela Leung,

University of California,

Los Angeles, United States

Maria Brito,

Icahn School of Medicine at

Mount Sinai, United States

${ }^{*}$ Correspondence:

Poupak Fallah

poupak@int.med.unipi.it

Specialty section:

This article was submitted to

Thyroid Endocrinology,

a section of the journal

Frontiers in Endocrinology

Received: 04 April 2017

Accepted: 21 September 2017

Published: 03 October 2017

Citation:

Fallahi P, Ruffilli I, Giuggioli D, Colaci M. Ferrari SM, Antonelli A and Ferri C (2017) Associations between

Systemic Sclerosis and

Thyroid Diseases.

Front. Endocrinol. 8:266.

doi: 10.3389/fendo.2017.00266
We have reviewed scientific literature about the association of systemic sclerosis (SSc) and thyroid disorders. A high incidence, and prevalence, of new cases of autoimmune thyroiditis (AT) and/or hypothyroidism have been shown in sclerodermic patients (overall in the female gender). An association among a Th1 immune-predominance, low vitamin D levels, and AT have been also shown in SSc patients. Cases of Graves' disease (GD) have been described in SSc patients, too, according with the higher prevalence of thyroid autoimmunity. It has been also shown a higher prevalence of papillary thyroid cancer (PTC), in association with AT, in SSc patients. However, in order to confirm results about GD and thyroid cancer, studies in larger number of patients with SSc are needed. During the follow-up of SSc patients it would be appropriate to monitor carefully their thyroid status. The abovementioned data strongly suggest a periodic thyroid function follow-up in female SSc patients [showing a borderline high (although in the normal range) thyroid-stimulating hormone level, antithyroid peroxidase antibody positivity, and a small thyroid with a hypoechoic pattern], and, when necessary, appropriate treatments. In conclusion, most of the studies show an association among SSc, AT, and hypothyroidism, such as an increased prevalence of TC overall in SSc patients with AT. Only few cases of GD have been also described in SSc.

Keywords: systemic sclerosis, autoimmune thyroiditis, hypothyroidism, Graves' disease, thyroid cancer, antithyroperoxidase antibodies, antithyroglobulin antibodies, CXCL10

\section{INTRODUCTION}

Systemic sclerosis (SSc) is a connective tissue disease characterized by degenerative microvascular phenomena and immune system activation, that lead to fibrosis of the skin and internal organs $(1,2)$. SSc is clinically a multifaceted disorder derived from different contributions of the abovementioned pathogenetic mechanisms, through a multistep process that causes various clinical phenotypes (3). SSc is a heterogeneous autoimmune disease which has defined by three hallmarks: small vessel vasculopathy, production of autoantibodies, and fibroblast dysfunction. The exact etiology of the disease remains unknown, due to the complex nature of the cellular signaling pathways involved. However, there is strong and consistent evidence that the innate system, in particular

\footnotetext{
Abbreviations: AbTg, antithyroglobulin antibodies; AbTPO, antithyroid peroxidase antibodies; AITD, autoimmune thyroid disorders; AT, autoimmune thyroiditis; ATA, antithyroid antibodies; dcSSC, diffuse cutaneous scleroderma; FT3, free triiodothyronine; FT4, free thyroxine; GD, Graves' disease; HC, healthy controls; HT, Hashimoto's thyroiditis; ISSc, limited cutaneous scleroderma; MCTD, mixed connective tissue disease; OA, osteoarthritis; OR, odds ratios; PM/DM, polymyositis/ dermatomyositis; PTC, papillary thyroid cancer; RA, rheumatoid arthritis; SLE, systemic lupus erythematosus; SS, Sjögren's syndrome; SSc, systemic sclerosis; TC, thyroid cancer; TSH, thyroid-stimulating hormone.
} 
toll-like receptor signaling, is contributing to the progression and perhaps onset of SSc (4).

Two overlapping SSc forms exist: (a) a limited cutaneous scleroderma (ISSc), confined to the skin of face, hands and feet and (b) a diffuse cutaneous scleroderma ( $\mathrm{dcSS} c$ ), extended over other areas of the skin, that can involve visceral organs, as kidneys, lungs, heart, and gastrointestinal tract. Patients affected by the limited form show a good prognosis, with a 10-year survival in about $75 \%$ of patients; however, about $10 \%$ of them develop pulmonary arterial hypertension after 15 years. Patients with dcSSc have a 10 -year survival of $55 \%$; death is commonly associated with pulmonary, heart, and kidney involvement.

The diagnosis is established on the basis of clinical suspicion, the presence of autoantibodies (in particular anticentromere and anti-scl70/antitopoisomerase antibodies) and quite rarely on biopsy. Regarding the antibodies, $90 \%$ of SSc patients have a detectable antinuclear antibody; anticentromere antibody is more frequent in 1SSc (80-90\%) compared to dcSSc (10\%), and anti-scl70 is more common in dcSSc (30-40\%) (5).

The American College of Rheumatology set the diagnostic criteria for scleroderma in 1980 (6).

Systemic sclerosis is associated with significant morbidity (including skin thickening, finger ulcers, joint contractures, pulmonary fibrosis and hypertension, chronic diarrhea, and renal failure) (7).

Systemic sclerosis patients have high rates of symptoms of depression, and SSc is associated with substantially reduced health-related quality of life (8).

Many studies show a high prevalence of autoimmune thyroid disorders (AITDs) in SSc patients; however, contradictory results have been reported too. Here, we review the scientific literature about the possible association of SSc with autoimmune thyroiditis (AT), Graves' disease (GD), and also thyroid cancer (TC).

\section{SSc AND AT}

After the initial case reports $(9,10)$, the association of SSc and AITD has been evaluated by many studies (Table 1).

A first systematic study (11) reviewed patients with fatal SSc about pathologic and serologic evidence of thyroid disorders. Histologic evidence of severe fibrosis of the thyroid was reported in $14 \%$ of 56 SSc cases (versus 2\% of age- and gender-matched control autopsy series). Among $27 \mathrm{SSc}$ patients in whom thyroidstimulating hormone (TSH) and free thyroid hormones were measured, 7 (26\%) were hypothyroid, and 9 had euthyroid sick syndrome. Hypothyroid patients had thyroid glands with fibrosis, but a few lymphocytic infiltration. However, 6/7 of hypothyroid patients had elevated levels of circulating antithyroglobulin antibodies (AbTg). These findings suggested a thyroid autoimmune process leading to gland fibrosis and hypothyroidism in severe SSc patients.

A second study (12) found decreased free thyroxine (FT4), decreased free triiodothyronine (FT3), and increased TSH in 42 SSc patients, versus age and gender controls. However, changes in FT4, FT3, and TSH were small with mean values within normal ranges, suggesting a subclinical thyroid dysfunction.

In a further study, $77 \mathrm{SSc}$ patients were evaluated by measurements of basal FT4, FT3, TSH, and the TSH response to thyrotropin-releasing hormone (13). Eight patients (10\%) were hypothyroid. Antithyroid antibodies (ATA) were present in four of eight $(50 \%)$ of the hypothyroid patients.

Among 39 SSc patients (14), 2 patients had clinical hypothyroidism, while 7 had subclinical hypothyroidism. On the whole

TABLE 1 | Prevalence of thyroid autoimmunity in SSc patients versus controls, in the published studies that included an internal control group.

\begin{tabular}{|c|c|c|c|c|c|}
\hline Reference & SSc patients (n) & AITD\% in SSc patients & Controls $(n)$ & AITD \% in controls & $P$ \\
\hline Shahin et al. (17) & 24 & $\begin{array}{l}\text { Serum levels of FT4 in patients were significantly } \\
\text { lower than in controls }(7.46 \pm 2.7 \text { for patients } \\
\text { versus } 10.5 \pm 1.8 \text { for controls with } P<0.001) \text {. } \\
\text { Of the } 24 \text { patients, } 8 \text { ( } 33.3 \%) \text { showed } \\
\text { hypothyroidism, evidenced by decreased FT4 } \\
\text { and increased TSH beyond normal ranges, } 5 / 14 \\
(32.7 \%) \text { with dSSc versus } 3 / 10(30 \%) \text { with ISSc } \\
(P=n s)\end{array}$ & 15 & & \\
\hline Innocencio et al. (18) & 25 & 52 & 113 & 3.5 & \\
\hline Antonelli et al. (20) & F 184; M 18 & F 107/77 (58\%); M 7/11 (39\%) & F 368; M 36 & $\begin{array}{c}\text { F 100/268 (27\%); M 2/34 } \\
(6 \%)\end{array}$ & F 0.0001; M 0.0019 \\
\hline Marasini et al. (21) & 79 & $\begin{array}{l}\text { AbTg in } 14 \% \text { of patients; AbTPO in } 23 \% \text { of } \\
\text { patients }\end{array}$ & $\begin{array}{l}81 \text { women with } \mathrm{OA} \\
\text { serving as controls }\end{array}$ & $\begin{array}{l}\text { AbTg in } 13 \% \text { of patients; } \\
\text { AbTPO in } 11 \% \text { of patients }\end{array}$ & $\begin{array}{l}\text { ns (for AbTg); } 0.057 \\
\text { (for AbTPO) }\end{array}$ \\
\hline
\end{tabular}

AbTg, antithyroglobulin antibodies; AbTPO, antithyroperoxidase antibodies; F, female; FT4, free thyroxine; M, male; ns, not significant; OA, osteoarthritis; TSH, thyroid-stimulating hormone. 
9/39 (23\%) of SSc patients were hypothyroid, and 4/9 (44\%) had positive ATA. Circulating AbTg and/or antimicrosomal antibodies were positive in $18 \%$ of the 39 patients.

A high prevalence of thyroid autoimmunity (15) was also observed in 43 Hungarian SSc patients. ATA were detected in 14 cases (33\%) [ 4 cases with AbTg, 11 with antithyroperoxidase antibodies (AbTPO), and 5 with antimicrosomal antibodies].

Antithyroglobulin antibodies and AbTPO antibodies, FT3, FT4 and TSH, and HLA-DR typing were carried out in 85 SSc Italian patients (16). AbTg and AbTPO antibodies were detected in 12\% $(10 / 85)$ and $19 \%(16 / 85)$ of patients. Two patients with ATA had clinical hypothyroidism and shared the HLA-DR3 allele. A higher frequency of the HLA-DR15 was shown in SSc subjects in the presence of AbTPO antibodies than in patients without AbTPO.

Twenty-three female patients with SSc (mean age $37.7 \pm 12.7$ ) were evaluated for thyroid dysfunctions versus 15 normal females as controls (17). Mean serum levels of FT4 in SSc patients were significantly lower than in controls $(7.46 \pm 2.7$ versus $10.47 \pm 2.5)$. Of the 24 patients, 8 (33.3\%) showed hypothyroidism (17).

Another Latin American study confirmed a high prevalence of thyroid autoantibodies in Brasilian patients with SSc (18).

In a large cohort study (19), 1,517 patients with rheumatoid arthritis (RA), systemic lupus erythematosus (SLE), SSc, Sjögren's syndrome (SS), mixed connective tissue disease (MCTD), and polymyositis/dermatomyositis (PM/DM) were evaluated for thyroid dysfunctions clinically and by imaging and fine-needle aspiration cytology [with respect to prevalence of GD or Hashimoto's thyroiditis (HT) in the general population]. HT was more common among MCTD, SS, and RA patients (21, 7, and 6\%, respectively) than GD $(2.5,3$, and $1.6 \%$, respectively). SLE, RA, SSc, MCTD, SS, and PM/DM had a higher prevalence for HT than the general population of 90-, 160-, 220-, 556-, 176-, and 69-fold, respectively, and for GD of 68-, 50-, 102-, 76-, 74-, and 37-fold, respectively.

A first study (20) aimed to assess the prevalence of thyroid disorders in SSc patients using a complete thyroid work-up, versus an internal appropriate control group. Two hundred two SSc patients versus 404 controls from the general population (matched by age and gender, with similar iodine intake) were evaluated for TSH, FT3, FT4, AbTg and AbTPO, thyroid ultrasonography and blood flow, and fine needle aspiration when needed. Odds ratios (OR) for female SSc versus controls subjects were significant for: clinical hypothyroidism, 14.5 (2.3-90.9); subclinical hypothyroidism, 3.2 (1.8-5.7); AbTPO positivity, 2.7 (1.8-4.1); thyroid hypoechoic pattern, 3.2 (2.2-4.7); thyroid autoimmunity, 3.7 (2.6-5.4); and thyroid volume $<6 \mathrm{~mL}, 1.8$ (1.2-2.7). OR for thyroid autoimmunity in male SSc patients versus control subjects was 10.8 (2.2-52.4). Female SSc patients had mean TSH levels significantly higher than control subjects, and female and male SSc patients had significantly higher AbTPO than controls. Three cases of GD in female SSc (versus zero in controls, $P<0.05$ ), and two of papillary thyroid cancer (PTC) were reported in SSc patients.

The authors suggested to test thyroid function, AbTPO, and ultrasonography and to assess the clinical profile of SSc patients. Thyroid function follow-up (and appropriate treatments) should be performed periodically in women, subjects with positive AbTPO and hypoechoic and small thyroid.
The abovementioned results were confirmed in another study (21) that evaluated thyroid function and autoantibodies, in 79 SSc women, versus 81 age-matched women with osteoarthritis (OA) as controls. Hypothyroidism was found in 16 of 79 (20\%) patients with SSc and in 9 of $81(11 \%)$ patients with OA. AbTPO were present in $23 \%$ SSc versus $11 \%$ controls. The risk of hypothyroidism was significantly higher in AbTPO-positive patients $(P<0.0001)$.

A cross-sectional study (22) of two convenience samples of patients with SSc, one in Canada and the other in Colombia, was performed. Among 719 patients, 273 (38\%) had at least one other autoimmune disease. Three hundred sixty-six autoimmune diseases were assessed, among which AITD (38\%), RA (21\%), SS (18\%), and primary biliary cirrhosis $(4 \%)$ were more frequent. Two hundred sixty patients (36\%) had first-degree relatives with at least one autoimmune disease [RA (18\%) and AITD (9\%) were the most common]. These results suggest that polyautoimmunity is frequent in SSc patients and autoimmune diseases cluster within families of these patients.

To determine (23) the ATA prevalence in a large SSc cohort and to verify whether they are associated with distinct clinical phenotypes, 138 SSc patients (46 with dSSc and 92 with 1SSc) and 100 healthy controls (HC) were tested for AbTg and AbTPO. A statistically significant increase of AbTPO was detected only in patients with $1 \mathrm{SSc}$ compared to HC (32.6 versus $14 \%, P=0.003$ ).

Two hundred ten SSc patients were evaluated in a Japanese study (24), that identified 30 patients with AITD (14.3\%), including 29 with HT (13.8\%) and 1 with GD (0.5\%).

A further study (25) first evaluated the incidence of new cases of clinical and subclinical thyroid dysfunction in SSc women versus controls from the same geographic area (matched by gender and age). SSc patients with thyroid dysfunction were excluded at the beginning, and then the manifestation of new cases of thyroid disorders was assessed in 179 patients and 179 matched controls, having a similar iodine intake (median follow-up: 94 months in controls; 73 months in SSc). An elevated incidence $(P<0.05)$ of new cases of hypothyroidism, thyroid dysfunction, AbTPO positivity, and hypoechoic thyroid, in SSc patients (15.5, 21,11 , and 14.6 of 1,000 patients per year; respectively) versus those in controls was observed. The onset of hypothyroidism was demonstrated (by logistic regression analysis) to be related to a borderline elevated initial TSH value, the presence of high AbTPO levels, and a hypoechoic and small thyroid in SSc patients.

A subsequent study (26) confirmed a high prevalence of subclinical hypothyroidism (8.5\%), overt hypothyroidism (1.9\%), subclinical hyperthyroidism (2.8\%), and overt hyperthyroidism $(0.9 \%)$ in SSc patients, and that a small thyroid volume $(<4.5 \mathrm{ml})$ was related to hypothyroidism.

Positive ATA titers were also observed in 27/86 SSc Polish (27) patients (31\%).

Recently, it has been also demonstrated that hypovitaminosis D was statistically associated with AT in SSc patients (28).

Conversely, a recent study (29) evaluated prospectively the prevalence of other autoimmune disorders in outpatient clinic in 3,069 consecutive patients with diagnosed chronic AT, with respect to two control groups (matched by age and gender): (a) a control group of 1,023 subjects, drawn out a random sample of the general 
population without thyroid disorders and (b) 1,023 patients with non-toxic multinodular goiter extracted from the same random sample of the general population, with similar iodine intake. The results of the study demonstrated a significant increase of the prevalence of SSc in AT patients (with respect to both controls).

Different studies demonstrate elevated circulating CXCL10 (Th1) and CCL2 (Th2) chemokines in SSc patients of newly diagnosis. Patients with a serious clinical phenotype, including the involvement of lung and kidney, have higher CXCL10. CXCL10 declines during the follow-up, while CCL2 does not change, suggesting the progress from a beginning Th1 inflammatory stage to a successive Th2 phase $(30,31)$.

Th1 lymphocytes, interferon- $\gamma$, and interferon- $\gamma$-dependent chemokines (CXCL9, CXCL10, CXCL11) play a pivotal role in AITD, that are Th1 immune-mediated autoimmune disorders, too (32-35). Newly diagnosed SSc patients have elevated circulating levels of CXCL10, but not of CCL2, in the presence of AT, indicating a predominance of the Th1 immune response in these patients (36).

To sum up, the abovementioned data show a high incidence, and prevalence, of new cases of AT, and hypothyroidism, in patients with SSc, suggesting that in SSc women, with a borderline high (though in the normal range) TSH level, in the presence of AbTPO, and a hypoechoic and small thyroid, it could be necessary to monitor periodically thyroid function.

\section{SSc AND GD}

A first anectodal study reported an association of SSc and GD in three cases (37). One case of GD was also observed among 210 SSc Japanese patients (0.5\%) (24). Graves' ophthalmopathy has been also occasionally reported in one SSc patient (38).

A significant number (3 cases) of GD in female SSc (3/202 versus $0 / 404$ controls, $P<0.05)$ was also observed in a case control study (20), with an internal appropriate control group.

On the whole the abovementioned studies suggest a higher prevalence of GD in SSc patients; nevertheless further studies, involving a larger number of SSc patients, are necessary to confirm this finding.

\section{SSc AND PTC}

Single cases of PTC in association with SSc were reported in several studies $(9,20,39-42)$.

However, more recently, the risk of TC in 327 unselected SSc patients with respect to two population-based, control groups was studied systematically (matched by age and gender; 654 subjects from an iodine-deficient area and 654 subjects from an iodine-sufficient area) (43).

Six subjects with PTC were detected among SSc patients, while only one case was observed in controls 1 , as well in controls 2 ( $P=0.007$, for both). In SSc, all patients with TC showed thyroid autoimmunity versus $40 \%$ of the other SSc patients $(P=0.001)(43)$.

These findings suggest the possibility of a increased prevalence of PTC in SSc patients with thyroid autoimmunity, however, larger cohorts are needed to elucidate this.

\section{CLINICAL ASPECTS OF SSC AND THYROID DISORDERS}

Several studies have evaluated a possible association among thyroid disorders and clinical findings of SSc, reporting different results.

It was initially reported that patients with hypothyroidism had more frequently subcutaneous calcinosis (11).

Hungarian SSc patients with AbTPO concentration tended to have secondary SS (15).

In a further study in female patients with SSc (duration $<3$ years), FT4 levels correlated significantly with Dlco\% $(r=+0.90, P<0.01)$, while in patients with SSc duration $>3$ years hypothyroidism correlated significantly with hand joint restriction of motion (17).

Seventeen SSc patients with high pulmonary systolic pressure $(>35 \mathrm{mmHg}$ ) were studied in another article (44). High pulmonary pressure in these SSc patients was not associated with the type of SSc, or age; however, five SSc patients $(12.5 \%)$ had alteration of the thyroid function (two cases of hypothyroidism, three of hyperthyroidism). The pulmonary pressure levels were higher in SSc patients with thyroid dysfunction, with respect to $\mathrm{SSc}$ in euthyroidism (40 versus $31 \mathrm{mmHg}$, respectively; $P<0.05$ ). Furthermore, hypothyroid SSc patients had higher pressure levels, with respect to hyperthyroid SSc (46 versus $37 \mathrm{mmHg}$, respectively), even if not significantly. The lack of significant differences in pressure levels between hypothyroid, or hyperthyroid SSc, and the low frequency of ATA in pulmonary hypertension associated with SSc, suggest a vasomotor role of thyroid hormones, rather than an autoimmune mechanism (44).

In Japanese female SSc patients, the prevalence of anticentromere antibody positivity, SS, and severe facial skin sclerosis was significantly prevalent in the presence of AITD (24).

A study found a statistically significant increase of AbTPO only in patients with 1SSc (but not in dSSc) compared to controls (32.6 versus $14 \%, P=0.003$ ) (23).

To sum up, most of the abovementioned studies did not observed any association among thyroid dysfunctions and/ or autoimmunity and features of SSc (clinical or serological) $(13,20,25,27)$.

\section{CONCLUSION}

Many studies have shown a high incidence, and/or prevalence, of new cases of AT, and hypothyroidism in SSc patients, especially in the female gender. An association among a Th1 predominance, low levels of vitamin $\mathrm{D}$, and AITDs has been also demonstrated in patients with SSc.

Few cases of GD have been also described in SSc patients, according with the higher prevalence of thyroid autoimmunity.

It has been also observed a higher prevalence of PTC, in association with AT, in SSc patients. However, in order to confirm results about GD and TC, studies in larger number of patients with SSc are needed.

To sum up, the abovementioned data strongly suggest that female SSc patients with a high risk (a borderline high though normal TSH value, the presence of AbTPO, and a hypoechoic 
and small thyroid) should be periodically monitored for thyroid function, and appropriate treatments when needed.

\section{AUTHOR CONTRIBUTIONS}

PF, IR, DG, MC, SMF, AA, and CF gave substantial contribution in the conception and design of the work, and in writing

\section{REFERENCES}

1. Ferri C, Sebastiani M, Lo Monaco A, Iudici M, Giuggioli D, Furini F, et al. Systemic sclerosis evolution of disease pathomorphosis and survival. Our experience on Italian patients' population and review of the literature. Autoimmun Rev (2014) 13:1026-34. doi:10.1016/j.autrev.2014.08.029

2. Barsotti S, Stagnaro C, d'Ascanio A, Della Rossa A. One year in review 2016: a systemic sclerosis. Clin Exp Rheumatol (2016) 34(Suppl 100):3-13.

3. Giuggioli D, Lumetti F, Colaci M, Fallahi P, Antonelli A, Ferri C. Rituximab in the treatment of patients with systemic sclerosis. Our experience and review of the literature. Autoimmun Rev (2015) 14:1072-8. doi:10.1016/j. autrev.2015.07.008

4. Dowson C, Simpson N, Duffy L, O'Reilly S. Innate immunity in systemic sclerosis. Curr Rheumatol Rep (2017) 19:2. doi:10.1007/s11926-017-0630-3

5. Jimenez SA, Derk CT. Following the molecular pathways toward an understanding of the pathogenesis of systemic sclerosis. Ann Intern Med (2004) 140:37-50. doi:10.7326/0003-4819-140-2-200401200-00013

6. Masi AT. Preliminary criteria for the classification of systemic sclerosis (scleroderma). Subcommittee for scleroderma criteria of the American Rheumatism Association Diagnostic and Therapeutic Criteria Committee. Arthritis Rheum (1980) 23:581-90. doi:10.1002/art.1780230510

7. Clements PJ, Roth MD, Elashoff R, Tashkin DP, Goldin J, Silver RM, et al. Scleroderma lung study (SLS): differences in the presentation and course of patients with limited versus diffuse systemic sclerosis. Ann Rheum Dis (2007) 66:1641-7. doi:10.1136/ard.2007.069518

8. Hudson M, Thombs BD, Steele R, Panopalis P, Newton E, Baron M, et al. Health-related quality of life in systemic sclerosis: a systematic review. Arthritis Rheum (2009) 61:1112-20. doi:10.1002/art.24676

9. Sánchez de Rivera JM, Barbado Hernández FJ, Vázquez Rodríguez JJ, Gil Aguado A, García Puig J, Viguer García-Moreno JM. [Scleroderma with traces of disseminated lupus erythematosus associated with Hashimoto's thyroiditis and papillary carcinoma of the thyroid gland (author's transl)]. Med Clin (Barc) (1979) 73:342-6.

10. Kaplan G, Vinceneux P, Bourgeois P, Kahn MF. [Gougerot-Sjögren syndrome, connective tissue diseases and autoimmune pathology]. Rev Rhum Mal Osteoartic (1977) 44:471-8.

11. Gordon MB, Klein I, Dekker A, Rodnan GP, Medsger TA Jr. Thyroid disease in progressive systemic sclerosis: increased frequency of glandular fibrosis and hypothyroidism. Ann Intern Med (1981) 95:431-5. doi:10.7326/0003-4819-95-4-431

12. Serup J, Hangdrup H. Thyroid hormones in generalized scleroderma. A controlled study. Acta Derm Venereol (1986) 66:35-8.

13. Kahl LE, Medsger TA Jr, Klein I. Prospective evaluation of thyroid function in patients with systemic sclerosis (scleroderma). J Rheumatol (1986) 13:103-7.

14. De Keyser L, Narhi DC, Furst DE, Huberman AK, Ross R, Clements J, et al. Thyroid dysfunction in a prospectively followed series of patients with progressive systemic sclerosis. J Endocrinol Invest (1990) 13:161-9. doi:10.1007/ BF03349530

15. Molnár I, Balázs C, Szabó E, Czirják L. Evaluation of thyroid function and anti-thyroid autoantibodies in systemic sclerosis. Acta Derm Venereol (1992) 72:112-4.

16. Molteni M, Barili M, Eisera N, Scrofani S, Mascagni B, Zulian C, et al. Antithyroid antibodies in Italian scleroderma patients: association of anti-thyroid peroxidase (anti-TPO) antibodies with HLA-DR15. Clin Exp Rheumatol (1997) 15:529-34.

17. Shahin AA, Abdoh S, Abdelrazik M. Prolactin and thyroid hormones in patients with systemic sclerosis: correlations with disease manifestations and activity. Z Rheumatol (2002) 61:703-9. doi:10.1007/s00393-002-0413-7 the article. AA and CF revised it critically for important intellectual content. PF, IR, DG, MC, SMF, AA, and CF gave the final approval of the version to be published. PF, IR, DG, MC, SMF, AA, and CF agreed to be accountable for all aspects of the work in ensuring that questions related to the accuracy or integrity of any part of the work are appropriately investigated and resolved.

18. Innocencio RM, Romaldini JH, Ward LS. High prevalence of thyroid autoantibodies in systemic sclerosis and rheumatoid arthritis but not in the antiphospholipid syndrome. Clin Rheumatol (2003) 22:494. doi:10.1007/ s10067-003-0803-5

19. Biró E, Szekanecz Z, Czirják L, Dankó K, Kiss E, Szabó NA, et al. Association of systemic and thyroid autoimmune diseases. Clin Rheumatol (2006) 25:240-5. doi:10.1007/s10067-005-1165-y

20. Antonelli A, Ferri C, Fallahi P, Cazzato M, Ferrari SM, Sebastiani M, et al. Clinical and subclinical autoimmune thyroid disorders in systemic sclerosis. Eur J Endocrinol (2007) 156:431-7. doi:10.1530/EJE-06-0591

21. Marasini B, Ferrari PA, Solaro N, Selmi C. Thyroid dysfunction in women with systemic sclerosis. Ann N Y Acad Sci (2007) 1108:305-11. doi:10.1196/ annals. 1422.032

22. Hudson M, Rojas-Villarraga A, Coral-Alvarado P, López-Guzmán S, Mantilla RD, Chalem $\mathrm{P}$, et al. Polyautoimmunity and familial autoimmunity in systemic sclerosis. J Autoimmun (2008) 31:156-9. doi:10.1016/j.jaut.2008.05.002

23. Danielides S, Mavragani CP, Katsakoulas I, Zintzaras E, Drosos AA, Vlachoyiannopoulos PG, et al. Increased prevalence of anti-thyroid antibodies in patients with limited scleroderma. Scand J Rheumatol (2011) 40:299-303. d oi:10.3109/03009742.2010.549837

24. Toki S, Motegi S, Yamada K, Uchiyama A, Ishikawa O. Demographic and clinical features of autoimmune thyroid disorder in Japanese patients with systemic sclerosis. J Dermatol (2014) 41:1053-7. doi:10.1111/1346-8138.12698

25. Antonelli A, Fallahi P, Ferrari SM, Mancusi C, Giuggioli D, Colaci M, et al. Incidence of thyroid disorders in systemic sclerosis: results from a longitudinal follow-up. Clin Endocrinol Metab (2013) 98:E1198-202. doi:10.1210/ jc. 2012-3583

26. Singh PK, Sharma SK, Sinha A, Dutta P. Can thyroid volume predict thyroid dysfunction in patients with systemic sclerosis? A prospective cross-sectional study from a tertiary care center in North West India. Clin Rheumatol (2016) 35:765-9. doi:10.1007/s10067-016-3209-x

27. Wielosz E, Majdan M, Koszarny A, Dryglewska M, Tabarkiewicz J. Presence of organ-specific antibodies in patients with systemic sclerosis. Pol Arch Med Wewn (2016) 126:862-9. doi:10.20452/pamw.3583

28. Giuggioli D, Colaci M, Cassone G, Fallahi P, Lumetti F, Spinella A, et al. Serum 25-OH vitamin D levels in systemic sclerosis: analysis of 140 patients and review of the literature. Clin Rheumatol (2017) 36:583-90. doi:10.1007/ s10067-016-3535-z

29. Fallahi P, Ferrari SM, Ruffilli I, Elia G, Biricotti M, Vita R, et al. The association of other autoimmune diseases in patients with autoimmune thyroiditis: review of the literature and report of a large series of patients. Autoimmun Rev (2016) 15:1125-8. doi:10.1016/j.autrev.2016.09.009

30. Antonelli A, Ferri C, Fallahi P, Ferrari SM, Giuggioli D, Colaci M, et al. CXCL10 (alpha) and CCL2 (beta) chemokines in systemic sclerosis - a longitudinal study. Rheumatology (Oxford) (2008) 47:45-9. doi:10.1093/rheumatology/ kem313

31. George PM, Oliver E, Dorfmuller P, Dubois OD, Reed DM, Kirkby NS, et al. Evidence for the involvement of type I interferon in pulmonary arterial hypertension. Circ Res (2014) 114:677-88. doi:10.1161/CIRCRESAHA. 114.302221

32. Antonelli A, Ferrari SM, Corrado A, Di Domenicantonio A, Fallahi P. Autoimmune thyroid disorders. Autoimmun Rev (2015) 14:174-80. doi:10.1016/j.autrev.2014.10.016

33. Antonelli A, Ferrari SM, Giuggioli D, Ferrannini E, Ferri C, Fallahi P. Chemokine (C-X-C motif) ligand (CXCL)10 in autoimmune diseases. Autoimmun Rev (2014) 13:272-80. doi:10.1016/j.autrev.2013.10.010

34. Antonelli A, Ferrari SM, Frascerra S, Pupilli C, Mancusi C, Metelli MR, et al. CXCL9 and CXCL11 chemokines modulation by peroxisome proliferator-activated 
receptor-alpha agonists secretion in Graves' and normal thyrocytes. JClin Endocrinol Metab (2010) 95:E413-20. doi:10.1210/jc.2010-0923

35. Ferrari SM, Elia G, Virili C, Centanni M, Antonelli A, Fallahi P. Systemic lupus erythematosus and thyroid autoimmunity. Front Endocrinol (2017) 8:138. doi:10.3389/fendo.2017.00138

36. Antonelli A, Ferri C, Fallahi P, Colaci M, Giuggioli D, Ferrari SM, et al. Th1 and Th2 chemokine serum levels in systemic sclerosis in the presence or absence of autoimmune thyroiditis. J Rheumatol (2008) 35:1809-11.

37. Nicholson D, White S, Lipson A, Jacobs RP, Borenstein DG. Progressive systemic sclerosis and Graves' disease. Report of three cases. Arch Intern Med (1986) 146:2350-2. doi:10.1001/archinte.1986.00360240064012

38. Wimmersberger Y, Zuercher D. Graves' disease associated with primary systemic sclerosis. Orbit (2009) 28:262-3. doi:10.3109/01676830903104637

39. Kaşifoğlu T, Yaşar Bilge Ş, Yıldız F, Özen G, Pehlivan Y, Yılmaz N, et al. Risk factors for malignancy in systemic sclerosis patients. Clin Rheumatol (2016) 35:1529-33. doi:10.1007/s10067-016-3288-8

40. Thongpooswan S, Tushabe R, Song J, Kim P, Abrudescu A. Mixed connective tissue disease and papillary thyroid cancer: a case report. Am J Case Rep (2015) 16:517-9. doi:10.12659/AJCR.894176

41. Kopeć-Medrek M, Kotulska A, Zycińska-Debska E, Widuchowska M, Kucharz EJ. Exacerbated course of atrophoderma of Pasini and Pierini in patient with papillary cancer of the thyroid gland. Wiad Lek (2010) 63:24-6.
42. Gokula RM, Gupta AK, Shirley SE, Coard K, Ramphal PS. Scleroderma with cardiac tamponade, hyperthyroidism and incidental papillary thyroid carcinoma. West Indian Med J (2002) 51:188-90.

43. Antonelli A, Ferri C, Ferrari SM, Di Domenicantonio A, Giuggioli D, Galleri D, et al. Increased risk of papillary thyroid cancer in systemic sclerosis associated with autoimmune thyroiditis. Rheumatology (Oxford) (2016) 55:480-4. doi:10.1093/rheumatology/kev358

44. Marasini B, Massarotti M, Cossutta R. Thyroid function, pulmonary arterial hypertension and scleroderma. Am J Med (2005) 118:322-3. doi:10.1016/j. amjmed.2004.09.020

Conflict of Interest Statement: The authors declare that the research was conducted in the absence of any commercial or financial relationships that could be construed as a potential conflict of interest.

Copyright $\odot 2017$ Fallahi, Ruffilli, Giuggioli, Colaci, Ferrari, Antonelli and Ferri. This is an open-access article distributed under the terms of the Creative Commons Attribution License (CC BY). The use, distribution or reproduction in other forums is permitted, provided the original author(s) or licensor are credited and that the original publication in this journal is cited, in accordance with accepted academic practice. No use, distribution or reproduction is permitted which does not comply with these terms. 